\title{
Amnestic MCI Patients' Perspectives on Volunteer Participation in a Research Context
}

\author{
Gwendolien Vanderschaeghe ${ }^{1^{*}}$, Jolien Schaeverbeke ${ }^{2,3}$, Rik Vandenberghe ${ }^{2,3,4}$ and Kris Dierickx ${ }^{1}$ \\ ${ }^{1}$ Department of Public Health and Primary Care, Centre for Biomedical Ethics and Law, KU Leuven, Belgium \\ ${ }^{2}$ Department of Neurosciences, Laboratory for Cognitive Neurology, KU Leuven, Belgium \\ ${ }^{3}$ Alzheimer Research Centre KU Leuven, Leuven Research Institute for Neurodegenerative Disorders, Belgium \\ ${ }^{4}$ Department of Neurology, University Hospitals Leuven, Belgium
}

"Corresponding author: Gwendolien Vanderschaeghe, Department of Public Health and Primary Care, Centre for Biomedical Ethics and Law, KU Leuven, Kapucijnenvoer 35 Blok D Box 7001, 3000 Leuven, Belgium, Tel: +3216 377629; E-mail: vanderschaeghe.gwendolien@gmail.com

Received date: May 03, 2017; Accepted date: May 22, 2017; Published date: May 25, 2017

Copyright: (c) 2017 Vanderschaeghe G. This is an open-access article distributed under the terms of the Creative Commons Attribution License, which permits unrestricted use, distribution, and reproduction in any medium, provided the original author and source are credited.

\begin{abstract}
Background: Inclusion of patients in research studies is immensely important when evaluating new biomarkers for Alzheimer's disease (AD) and when the efficacy of possible treatment options is under trial investigation. If medical treatment is to advance in slowing the progression of $A D$, or even preventing it, voluntary participation of patients is not only important but also their reliable participation is key. To get closer in achieving this goal, researchers need to understand better what motivates research participants to enroll in a clinical trial and get insight into participants' expectations about their participation. Furthermore, what researchers perceive as benefits and risks of a study can differ from participants' view. This difference could lead to a situation in which researchers recruit fewer subjects than they expected or result in unreliable research subject participation.
\end{abstract}

Method: We conducted semi-structured in-depth interviews in 38 patients with amnestic mild cognitive impairment (aMCl) as part of a clinical trial (EUDRACT no. 2013-004671-12) on the predictive value of biomarkers for AD. Patients had the option of receiving their Individual Research Results (IRR; visual binary read amyloid PET results). In this study, the motivations and perceived advantages and disadvantages of trial participation were investigated from the patients' perspective. Before deciding to participate, patients received an information brochure describing the possible benefits and risks of participation.

Results: The two most frequently mentioned reasons for volunteering for the trial were to contribute to scientific progress and to receive their IRRs. Participating to ameliorate scientific progress was not solely motivated by altruistic reasons; it was mostly mentioned along with the possibility of receiving a valuable result about their health condition, suggesting that self-interest also motivated patients to participate. The two most frequently mentioned disadvantages were the possible risks related to being subjected to invasive medical procedures and that volunteering was considered to be time consuming. Most patients felt that their partner and children supported their decision to enroll in the biomarker study.

Conclusion: aMCI patients have several reasons for wanting to volunteer in a clinical trial, with the option of IRR disclosure being the primary motivation for enrolling. Most of our patients felt that family members supported their decision to volunteer. However, researchers need to be cautious when recruiting subjects for clinical trials by ensuring that they truly desire to participate in the study and those family members are not coercing them into doing so. In addition, what the information brochure mentions as possible benefits and risks of trial participation was not always perceived similarly by patients.

Keywords: Ethics; Amnestic MCI; Clinical trial; Qualitative research; Participation reasons; Biomarker; Amyloid PET scan; Belgium

\section{Introduction}

Reliable participation from volunteer subjects is of utter importance to draw firm conclusions from research data. This also applies to the study of Alzheimer's disease (AD), which often involves participation of healthy adults and patients with mild cognitive impairment (MCI). A common theme across $\mathrm{AD}$ studies relates to identifying biomarkers for accurate diagnosis and track disease progression. This is especially important because there is an urgent need to detect the disease at an earlier stage and to identify those subjects who are most likely to exhibit rapid disease progression in order to reduce healthcare burden and economic costs. Due to the difficulty in clearly recognizing the first symptoms of $\mathrm{AD}$ in a clinical setting, only an estimated one-half of all cases are diagnosed [1,2]. Therefore, diagnostic trials are important for developing and validating new biological markers for AD either as diagnostic or predictive tool. These markers could also be used in therapeutic clinical trials to evaluate new potential treatments. It has been a challenge to prevent and slow down disease progression in $\mathrm{AD}$ because of the limited efficacy of current treatment options. Researchers need to explore what motivates individuals to enroll in a clinical trial and what participants perceive as trial benefits and risks, and then leverage these vis-à-vis participation to stimulate and achieve reliable research subject participation [3]. 
The prevailing literature on motivations to participate in research studies concerns other research fields, such as genetics-related studies $[3,4]$. One focus group study by Lawrence et al. explored patient and carer views specifically on this topic [5]. However, their results were based on two different perspectives (patients and carer), which were conflated. Few studies have been done on participants' motivations in $\mathrm{AD}$ trials. There is therefore a need for more in-depth research on $\mathrm{AD}$ study subjects' motivations and perceptions of possible advantages and disadvantages of trial participation.

The current study is part of the BioAdaptAD study on the predictive value of biomarkers in a cohort of amnestic MCI (aMCI) patients who could choose to know their IRRs (visual binary read of amyloid PET scan). Findings from the participants' perspectives toward amyloid PET disclosure are reported previously in Vanderschaeghe et al. [6]. In this study, we examined subjects' motivations and their perceived disadvantages of participating in a clinical trial by means of semistructured interviews.

\section{Methods}

\section{Recruitment}

Recruitment of subjects took place between June 2015 and June 2016 after approval of the study by the Ethics Committee, University Hospitals Leuven. All participants provided written informed consent in accordance with the Declaration of Helsinki.

The study cohort consisted of a consecutive series of aMCI patients [7] recruited via the memory clinic of the University Hospitals Leuven. The interview was part of a substudy of the BioAdaptAD study, an investigator-driven longitudinal study of aMCI patients. The primary objective of the BioAdaptAD study (EUDRACT no. 2013-004671-12) is to evaluate the predictive value of baseline amyloid biomarker measurements for tracking longitudinal cognitive change over a twoyear period.

According to Standard Operating Procedures, on occasion of a follow-up outpatient visit to the memory clinic, the patient was informed about the possibility to participate in a trial and the purpose and content of the trial was introduced to the patient by the physician and by the researcher (JS). The information brochure and the informed consent form was given to the patient so that the patient could discuss this further with anybody of their choice at home. One week later the patient was recontacted by phone to ask whether they had decided to volunteer in the clinical trial.

When candidate subjects met the inclusion criteria (Appendix 1) of the BioAdaptAD study and decided to enroll in the trial, they were given an additional option to participate in a substudy investigating participant motivations for choosing to enroll in a clinical trial, their rationale for opting for their amyloid PET scan result and the ethical challenges associated with it. As mentioned in the introduction, the findings from the amyloid PET disclosure have been reported in another publication [6]. The same cohort population contributed to the current study. We conducted semi-structured interviews with participants who agreed to take part in the substudy in order to better understand their motivations, opinions, and experiences about their trial participation.

Before the start of the substudy, an informed consent brochure about the interviews was given to candidate subjects. The content of this brochure was based on the E6 Guideline of Good Clinical Practice
(GCP) [8] and contained background information about the substudy, study objectives, the interview process, and research subjects' rights. Before the scheduled interview, the interviewer orally repeated the content of the informed consent brochure and asked the candidate whether they had any further questions. If they hesitated or had doubts about participating, the interview was re-scheduled for a later time in order to give the candidate sufficient time to decide.

\section{Data collection and analysis}

The interview guide was developed by GV, KD, and RV, and its content was based on findings in the literature on the topic of IRR. The first two interviews constituted of a pilot study, which was used to evaluate the interview guide. The interview questions covered three content areas. The first part of the interview consisted of questions intended to better understand how patients describe and experience their current memory problems. The second part consisted of openended questions about why they chose to participate and expectations they had about being part of a clinical research trial. The third part consisted of hypothetical questions about how the participant thinks he would respond to a set of possible situations. Several interview techniques were used, such as rephrasing part of the participant's answer, asking yes or no questions, to briefly checking whether the interviewer understood the participant's answer correctly. Supplementary questions were also asked to get more in-depth information from the participant.

After a short introduction of the research set-up and what to expect in the interviews, patients were invited to sign the informed consent form. Patients also completed the sociodemographic information form. Completion of the latter form indicated that the patient understood that the study results and interview records would remain confidential, that participation in the interview was voluntary, and that it would have no impact on participation in the general study or on any other medical intervention the patient might undergo in a clinical context. Patients were informed that study results would be published in a scientific journal and that a lay description of results from the interviews would be provided to them after completion of the study.

The interviews were recorded on tape with the consent of the interviewee. A mixed-methods approach was used to analyze the interviews (1). Transcripts were analyzed using QSR International's Nvivo 11 software, and analysis was performed according to qualitative conventional content analysis methodology $[9,10]$. In the first phase, the interviewer $(\mathrm{GV})$ coded the interviews at three separate time points, with an interval of a few weeks between each coding session. To protect against bias, in the second analysis phase, we had five interviews independently coded by a second researcher. In the final phase, we compared the codes assigned by the interviewer and those done by the independent researcher (KD), working to reach a consensus on the final codes to be used (2). In addition, we used a quantitative approach to analyze the reasons, benefits, and disadvantages of receiving an IRR, as provided by the participants.

Interviews were conducted in Dutch, with the exception of one, which was conducted in English. For this interview, the patient preferred to speak in his native language, English. Quotations of patients presented here have been translated into English. 
Citation: Vanderschaeghe G, SchaeverbekeJ, Vandenberghe R, Dierickx K (2017) Amnestic MCl Patients' Perspectives on Volunteer

Page 3 of 8

\section{Results}

\section{Study population}

Sixty-seven patients were invited to participate in the BioADadapt study; 26 of them decided not to volunteer, and three patients quitted the study after the first neuropsychological screening visit. Most of these individuals did not provide a reason or explanation for this decision, yet some referred to the burden of caring for a needy partner or to a lack of motivation. For the three patients who dropped out of the study, the following three reasons were mentioned; a lack of motivation, a refusing attitude from the patient his partner and an inability to schedule a new appointment that best suited the patient. This resulted in a study population that consisted of 38 aMCI patients who met the inclusion criteria of the study (Appendix 1). Table 1 summarizes the demographic information of participating aMCI patients and their scores on tests of the neuropsychological evaluation.

\begin{tabular}{|c|c|}
\hline Characteristics & Number \\
\hline $\begin{array}{l}\text { Mean Age } \\
71 \pm 6.5 \text { y (range: } 55-83 \text { y) }\end{array}$ & - \\
\hline $\begin{array}{l}\text { Gender } \\
\text { Male } \\
\text { Female }\end{array}$ & $\begin{array}{l}22 \\
16\end{array}$ \\
\hline $\begin{array}{l}\text { Highest educational level attained } \\
\text { Primary school } \\
\text { Secondary school } \\
\text { Professional bachelor } \\
\text { Academic master }\end{array}$ & $\begin{array}{l}7 \\
17 \\
6 \\
8\end{array}$ \\
\hline $\begin{array}{l}\text { Marital status } \\
\text { Married } \\
\text { Widow/widower } \\
\text { Divorced }\end{array}$ & $\begin{array}{l}30 \\
4 \\
4\end{array}$ \\
\hline$\geq 1$ Children & 36 \\
\hline Neuropsychological evaluation & Mean score \\
\hline $\begin{array}{l}\text { Global Clinical Dementia Rating } \\
\text { scale }\end{array}$ & 0.5 \\
\hline MMSE (/30) & 27.8 (range: $25-30$ ) \\
\hline AVLT: Total Learning (/75) & $36.2 \pm 10.2$ \\
\hline AVLT: long term $\%$ recall & $61.7 \% \pm 29.6 \%$ \\
\hline
\end{tabular}

Table 1: Demographic characteristics and neuropsychological evaluation scores of the study population of aMCI patients (Memory test results: MMSE = Mini mental state examination, AVLT = Rey Auditory Verbal Learning (AVLT) scores for Percentage Delayed Recall (\%DR, score on 30 min delayed recall (/15) divided by the score on last trial (/15)) and Total learning (TL,/75).

\section{Patients' motivation for enrolling in a clinical trial}

We investigated what motivates participants to volunteer in a research study. In other words, what specific reasons do aMCI patients cite for enrolling in a clinical trial? From the interview transcripts, we identified seven main reasons for enrollment in this trial (Figure 1). Some participants mentioned two or three of these reasons. 


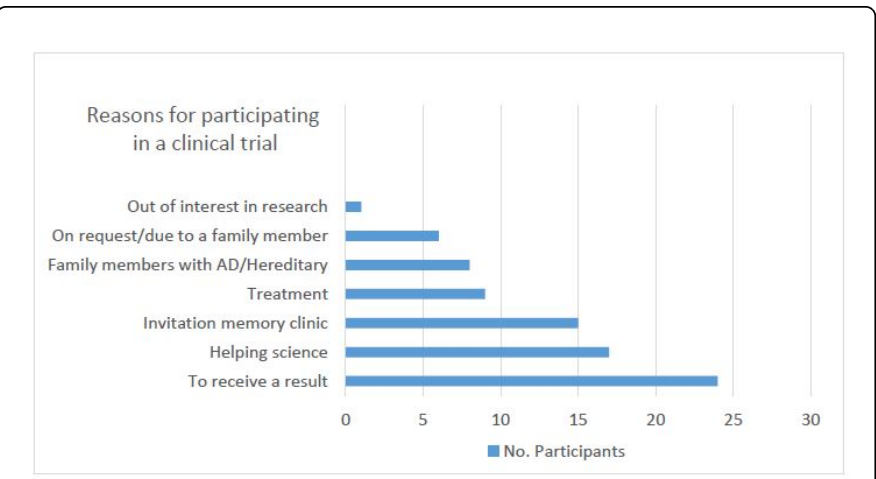

Figure 1: Specific reasons patients cited for wanting to participate in a clinical trial.

These seven reasons will be considered in more detail in the following section. We present quotes of the patients that serve to illuminate these reasons. The first reason was having an interest in research. One participant mentioned how he has always been interested in research. For him, this interest alone provided him with enough incentive to volunteer in the clinical study.

The second reason cited by some participants for enrolling in the trial was because family members encouraged them to do so. Mostly the participant's partner, and often together with their children, noticed increasing forgetfulness in the participant. Family members not only advised the participants to go to the memory clinic for medical advice but also prompted them to volunteer in this trial.

"But I wouldn't have come on my own, but my husband wanted me to. But yes, he perhaps experiences it better [...]. But I think my memory is not the same as in the past because I used to know everything, and now I have to look up (things) sometimes." (Woman, 72 years old).

The third reason for enrolling in the trial was having a family member with AD. Of all participants, one-quarter had one or more family members who were diagnosed with some form of dementia or likely $\mathrm{AD}$. These participants wanted to get an idea whether they too might develop the condition. Since they have witnessed firsthand how $\mathrm{AD}$ affected their relatives, they are well aware of its impact, and this provided them with the motivation to volunteer and to undergo testing for AD. Some participants mentioned that dementia was highly prevalent in their family, indicating that this could be hereditary. For some participants the occurrence of the disease within their family also led to fear:

"Because in my family, there are many demented. So that scares me. It really scares me." (Woman, 72 years old).

The fourth reason was the desire to receive treatment. Many participants were hoping to receive medication that would delay or slow down the progression of their disease. One participant even referred to the information brochure about the clinical trial, stating that in the event of a positive amyloid PET scan result, cholinesterase inhibitors would be administered. Although most participants were aware that at present $\mathrm{AD}$ is incurable, they did have some expectations about treatment:
"I expect that they can stop this. [...] that I can get a treatment for that. So that I won't end up like my brother." (Man, 74 years old).

The fifth reason was being invited by memory clinic personnel to participate in the trial after their recent follow-up at the memory clinic or during their first clinical consultation. Two participants mentioned that they discussed the invitation with their general practitioner (GP), and one participant indicated that his GP advised him to go the memory clinic, where he was invited to participate.

The sixth reason for volunteering for the trial was the desire to contribute to science and medicine. Some participants indicated that without their participation, research could not make medical progress. Another participant mentioned that, due to his current memory complaints, he might be an interesting case for scientists to investigate. However, most participants stated they enrolled in the trial not only to contribute to scientific research but also to find out what is going on with their health. We observed that the participants did not volunteer for purely altruistic reasons, as shown in the following quote: "Yes absolutely, it is not just for myself, well a big part for myself (laughs)." (Woman, 67 years old).

The seventh and most frequently mentioned reason for participating in the trial was to receive a medical test result. For many of the participants, it was very important to find out what caused their subtle memory complaints. One participant stated that $\mathrm{AD}$ is a common topic of discussion today, and just hearing discussions about $\mathrm{AD}$ can cause one to wonder whether one will develop $\mathrm{AD}$ in the future.

"[...] But since this has been in my head for many years, I think it's important to know, because you think about that a lot. There is a lot, a lot spoken and said about (AD) and so I want to know it." (Woman, 69 years old).

Some participants stated that, by participating in the trial, they were hoping to receive more information about their current health situation, and that this in turn, would help them understand more about their medical health situation.

"[...] I think that I will learn something more. And I want to know where I stand. What it is exactly." (Woman, 68 years old).

"Because again it was to find out why and at the moment, $[\ldots]$ you know, at least if you know the amount of amyloids there (is) or not, that is, you are sure or relatively sure." (Man, 66 years old).

\section{Perceived clinical trial advantages}

After exploring what motivated participants to enroll in a clinical trial, we investigated whether participants perceived any advantages or benefits to participating in a clinical trial. First, a minority of participants spontaneously reported that there were no advantages to volunteering in a clinical trial. However, two participants subtly qualified their answer without hesitation:

"No, not directly. No, I don't see... except with the results to know what's going on. For the rest, I personally do not see any real advantages." (Man, 73 years old).

However, other participants did perceive certain advantages when participating in a trial, as described next. 


\title{
Clinical trial advantages
}

\author{
No advantages \\ Medicine/treatment \\ Contribution to science \\ Knowing your result
}
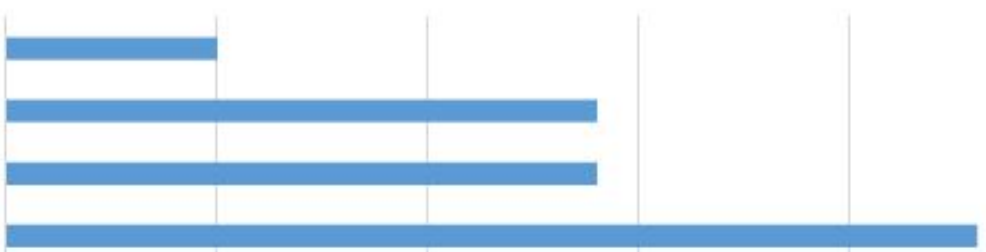

0

10

15

20

No. Participants

Figure 2: Clinical trial advantages perceived by participants.

Participants mentioned three advantages associated with participating in a clinical trial. First, medication and treatment were perceived as an advantage. When participants used the word "medication," they were referring to medicine that could delay or stop the disease. When participants used the word "treatment," this suggested a broader connotation and did not solely refer to medication use (Figure 2). Participants also viewed "treatment" as the monitoring or following up of the disease. Some of the participants were eager to learn their test results and believed that the sooner they received them the sooner treatment could start:

"[...] If they find something, they can help me sooner, so I can be independent for as long as possible. I have seen it with my mother. She has been taking pills for two years now. Now it doesn't start to work that well, but it has still kept her at home for two, three more years. And yes, yes, I think that is certainly an advantage for me." (Woman, 61 years old).

One participant stated that it is better to participate, because if something were wrong with her health, she at least had the option to receive treatment. She described her feelings about participating as follows:

[...] so participating is always the best option, it is the best chance I have." (Woman, 63 years old).

The second advantage of participating in a clinical trial that was mentioned, was to help science, which was also one of the reasons participants gave for participating. For some participants, the idea of being able to assist in advancing science not only served as their motivation to participate in the trial, but also as an advantage that could lead to medical improvements. Other participants provided a more nuanced response, stating that their participation would mainly benefit themselves, but that helping to advance science is a positive consequence. This sentiment is reflected in the following quote:

"[...] Yes, to help others, but I say it again. [...] I'll be straightforward with you, I'm quite selfish. I am trying to save my own skin here [...] I put it quite bluntly, but you can understand me." (Woman, 63 years old).

The third advantage of participating in a clinical trial was that participants would know their test result. Some participants expected to receive all information related to their health status:
"Ah yes, because you'll know everything [...], when something's wrong." (Woman, 76 years old).

Other participants expected to receive more information than what they believed they would receive during a regular doctor's office visit:

"I don't know if, well, with a normal diagnosis that it is always very fast. And the professor take one quick look (at you), and then he is back off again. (In a clinical trial) you'll receive more information." (Woman, 66 years old).

This quote is consistent with the experience of one participant, who noted that clinicians were often under time pressure.

\section{Perceived clinical trial disadvantages}

We also sought to determine whether participants perceived any disadvantages related to participating in a clinical trial. Eighteen out of 38 participants spontaneously reported that there were no disadvantages related to participating in a trial. To explore the possibility that these participants were hiding their true feelings and were just providing socially desirable answers, the interviewer asked them additional auxiliary questions. After this line of questioning, we were able to determine that these participants felt that the advantages of participating in the trial outweighed any possible, albeit minor, disadvantages. The frequency of possible disadvantages perceived by the participants is shown in Figure 3.

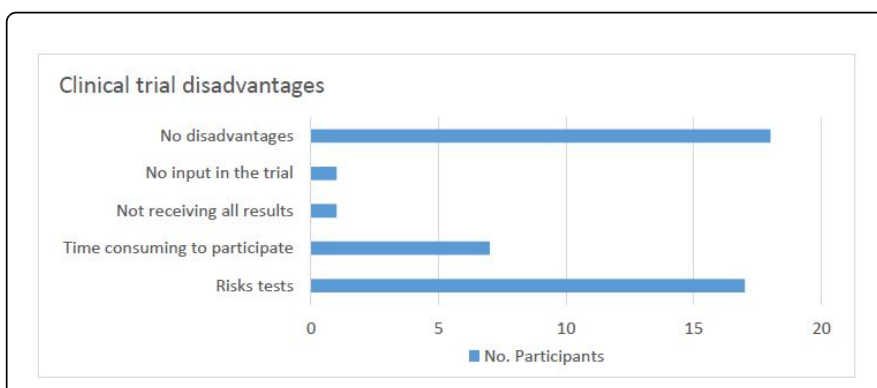

Figure 3: Clinical trial disadvantages perceived by the participants. 
The participants mentioned four disadvantages of participating in the trial. The first was that participants did not have any influence on the design of the clinical trial. One participant stated:

"But the question is: you have no input into the trial. So, no, I don't like being involved in things that I cannot control." (Man, 66 years old)

The same participant brought up another disadvantage: Not receiving all his personal data from this trial.

The third disadvantage was that trial participation is too time consuming. Participants viewed the trial as being too time consuming, because they had to visit the hospital several times in order to undergo a series of trial-related tests, such as neuropsychological investigation, MRI, PET scan, lumbar puncture (LP), and interviews. Most participants viewed these tests as only a minor inconvenience.

The fourth and last disadvantage mentioned was that trial participation is associated with certain risks. Most of the participants were aware of the risks involved with LPs and with amyloid PET scans, which require participants to receive an injection of a radioactive tracer. One participant stated that healthy people should not participate in a trial because of the possibility of exposing themselves to certain unnecessary risks:

"Well, it's a bit like, it's a simple lumbar puncture, but there are lots of ways. Just going to a hospital exposes you to more germs. And then, having a small procedure could be risky. Which, you know, I think it's different when you know there's something wrong with you and they say: 'Well, we need to examine you more thoroughly.' That's different than having to put yourself at risk if you don't need to." (Man, 66 years old).

For a few of the participants who mentioned possible risks as a disadvantage to trial participation, it was not very clear to them how these risks could affect them. Others checked the information brochure, asked questions, and informed themselves about potential risks by asking others, such as their GP, for advice. Risks associated with the trial sometimes caused participants to question whether they had made the right choice. One participant, a 66-year-old woman, stated that the LP "has made me think twice [...]" about participating in the trial. Although most participants were concerned about the possible risks of participating, other participants immediately downplayed the risks, indicating that they viewed these to be minimal compared to those associated with more invasive trials or experimental trials that test medications. Most participants trusted the innovative techniques and the capabilities of the researchers, which contributed to the view that the risks were minimal. However, one participant did question the capabilities of some researchers. This particular participant demanded that his LP would be performed by professional healthcare staff, not by an intern. In general, only a minority of the participants expressed slight hesitation about some of the procedures associated with the trial. This was because a previous LP did not go well for them or because they were afraid of the pain might be caused by possible complications.

\section{Family support}

The option of participating in a trial was discussed by most participants in advance with their partner, and often with their children as well. This situation depended on their familial background, as some participants were divorced, widowed, or had little contact with their children and/or other family members. There were some participants who did not discuss this with their partner or children, since they believed it was a personal decision.

Most participants felt that their partner supported their choice to participate in a trial and their decision to know their IRR. One participant rarely discussed trial participation with his wife, and it bothered him that she rushed him before the interviews, so they could leave on time and do other things. He found this to be difficult. $\mathrm{He}$ stated that his wife found it unpleasant to join him when he had to be at the hospital, yet she wanted to do all of this for him. Another participant also experienced such mixed messages from his wife:

"Yes and no, in a sense, she thinks there's nothing wrong with me. You know, when we say that there are two types of people: people who want to know, and people who are the complete opposite." (Man, 66 years old).

The last reason mentioned by patients revealed two other findings in our study. First, participants were not always certain whether their partner would volunteer in a trial and whether they would opt to know their IRR. They came to these conclusions because they believed that their partner would find it emotionally difficult to cope with bad news, had the disposition of not easily seeking medical advice or help, or had a resigned attitude: "Whatever happens, happens." Second, some participants indicated that when they were talking to others (partner, family members, and close friends) about their clinical trial participation, they frequently received the following reaction: "Why are you participating? There is nothing wrong with you?" This reaction was often difficult for the participant to accept because they felt that their memory problems were minimized or perceived as symptoms of normal aging.

\section{Discussion}

The current study aimed to gain insight into what motivates research participants to enroll in a clinical trial and their expectations about trial participation and complements an earlier report on amyloid PET disclosure [6]. The major strength of this qualitative research is the in-depth interviews with patients, as these face-to-face interviews provide researchers with the opportunity to delve into subjects' answers in-depth, clarify them, and check their opinions. This resulted in concrete motivational reasons (e.g. to receive a result, helping science, based on invitation of memory clinic, and so on) and perceived trial advantages (e.g. to know your result, treatment, and so on) and disadvantages (e.g. risks when undergoing tests, time consuming to participate, and so on) as mentioned by our study population. Based on our study results, we will comment and raise additional thoughts on five findings;

\section{Individual research results}

Most of the participants believed that it was common sense that they would receive their research results, since they participated in a clinical trial. In other words, they had a sense of ownership of any results that derived from their volunteer participation. This finding highlights the difficulties some participants had in understanding the differences between a clinical and research setting [11,12]. For example, most research subjects may be unaware that the main goal of research is to produce new general knowledge in a particular field of inquiry, not to produce and return individual bits of knowledge to a research participant $[13,14]$. This blurring of the boundary between clinical practice and research in the minds of research participants is often mentioned in the literature as an argument against the disclosure 
of these results [11-15]. Yet, there are also counter-arguments, such as the return of the IRRs would make research more transparent for society, who ultimately benefits and often funds the research [16]. Transparency may enhance public understanding and increase participants' willingness to contribute to future research studies [11,15-17]. However, it is understandable that participants perceive the disclosure of the IRR as common sense and that there is a blurring of the boundary between clinical care and the research setting. This may be due to the many clinical therapeutic trials where the line between research and clinical care is often blurred and in which, for example, a positive amyloid PET scan result is part of the inclusion criteria. In this case, participants become aware-although maybe implicitly_of their research result once they are accepted into the therapeutic clinical trial. This is different with regard to a non-disclosure policy in the research setting, which is not linked to therapeutic clinical trials. The implicit disclosure in therapeutic trials also raises several ethical concerns, as these results are usually not actively disclosed. For example, what if the participant is not aware of this implicit disclosure and does not wish to know the result? Is the implicit disclosure of a result accurately understood by the participant? How do researchers and clinicians handle follow-up, counseling and guidance after an implicit disclosure of a positive amyloid PET scan result to the participant?

\section{Limited efficacy of current treatment options}

We hypothesized that participants would mention the limited efficacy of current treatment options as a disadvantage of trial participation as this is often described as an ethical issue in the available literature on $\mathrm{AD}[18,19]$. As of yet, there is no treatment available to prevent or cure Alzheimer's Disease [20]. There is the possibility to use drugs, such as acetylcholinesterase inhibitors (AChE-1) and memantine which can provide symptomatic relief, delay progression and possibly improve the quality of life of the patient [21].

When we asked participants whether limited efficacy of current treatment options is a disadvantage, they stated that they already knew before the start of the study no cure was available or that available medication could only delay the progression of the disease. They perceived the latter as a better option than receiving no medication at all. Most participants maintained strong hope that in the near future this will change and that history has shown how medical research rapidly evolves in the direction of providing new curative medicines.

\section{Risks related to tests}

In the present study, the majority of participants viewed the risks associated with certain tests, such as LP (headache, pain) and amyloid PET scan (radiation exposure), as a disadvantage of their trial participation. To address such concerns, the guidelines of GCP require that all possible risks are explained in the research study information brochure and that the researcher must clearly explain the study protocol to potential research participants [8]. As indicated in the results, a minority of our participants mentioned the possibility of risks, yet stated that it was not very clear what these risks could imply for them. This finding reveals that it is difficult to ensure that all participants will clearly understand all aspects of a trial that may or may not affect them as described in the information brochure. This finding underscores that obtaining informed consent from research participants should be an active information process, during which the researcher devotes sufficient time to explain the study and informed consent in an understandable manner. Although we are aware that this is a time-consuming process for the researcher, our study findings indicate how necessary and beneficial this process is for the participant. In addition, if participants clearly know and understand what the clinical trial procedure implies, what they can expect, and what risks they might face, this may contribute to a reduction in dropouts through the course of the trial.

\section{Trial participation is time consuming}

In our study, we tried to conduct several tests in one visit at the hospital to maximize test opportunities and to minimize the burden for the patient by doing too many different visits with single tests. Yet, one in five participants still perceived that their participation was time consuming, although this was considered a minor disadvantage. A similar finding was reported by the clinical study of Christensen et al. [22]. They indicated that the desire to contribute to $\mathrm{AD}$ research was rated highly and seen as a benefit by participants, but after completing a 12-month follow-up and after receiving their ApoE status, their rating was lower when asked about this desire to participate in research [22]. The authors stated that it is possible that study participants' initial eagerness at enrollment became tempered somewhat after realizing the many practicalities of research participation, such as administration tasks and regular follow-ups [22]. To overcome this problem, two practical steps seem to be important for researchers to take: (1) Before and throughout the study, clearly explain to the participant what their participation consists of, what will happen, and how long approximately each visit will be (2). Cluster together as many tests as is feasible during each visit to the hospital. Doing this may help avoid subject dropout through the course of the trial. However, certain questions still need to be answered: Is it practically feasible to carry out several tests in one hospital visit? Will combining tests in one clinical visit influence or bias the results? Will a longer session with multiple tests be too burdensome; is it bearable for the participant?

\section{Family support}

Finally, this study demonstrates that a majority of participants feel supported and influenced by their family members regarding their trial participation. Although family support is a positive finding, caution is warranted in order to avoid a situation in which participants feel pressured by their family members to volunteer in a trial. Voluntary participation is also a requirement in the guidelines of GCP [8].

\section{Limitations}

One limitation is that the views expressed here are based on a relatively small population of aMCI patients in Belgium. This potentially limits the generalizability of our findings to other research contexts and other study populations. Different findings might emerge when an investigation takes place in a different country and when testing a different population, such as healthy elderly volunteers screened for preclinical AD or patients with mild cognitive complaints who have not undertaken steps to receive medical consult at the memory clinic. In addition, the interviews were part of a nontherapeutic trial. Different reasons for volunteering may emerge as part of a therapeutic clinical trial. Also, some individuals were given the option to enroll in a clinical trial, yet were not willing to participate. Some reasons were known, yet we do not have insight in all reasons of individuals who declined the possibility to volunteer. Future research could focus on the hesitation or refusal reasons from individuals who wish not to enroll in a clinical trial. Lastly, the interviews took place in Dutch, and used quotes were translated in English, which means that 
some of the interviewee's nuances may be lost. Despite these limitations, we believe that the present findings are an important first step in arriving at a better understanding of research subjects' motivations, will be useful beyond the context of this $\mathrm{AD}$ biomarker research, and will guide the design of future clinical trials.

\section{Conclusions}

Overall, aMCI participants have definite motivations for participation in a clinical trial and perceptions of what constitutes advantages and disadvantages of trial participation. The two most frequently mentioned reasons for volunteering in a trial were to contribute to science and to receive an IRR. The wish to make a contribution to science was not based purely on altruistic sentiments, but was a consequence of the participants' primary motivation of possibly receiving medical test results. Participants perceived several disadvantages of trial participation. The two most frequently mentioned disadvantages were the possible risks related to certain tests done in the trial and the fact that volunteering was time consuming. The latter indicates that researchers might consider reducing the number of hospital visits perhaps by clustering several tests within one visit, as far as practically feasible and to the degree that this would not impact on data quality. Most participants felt that their partner and/or children supported their decision to enroll in this study. However, researchers need to be cautious when recruiting participants for clinical trials by ensuring that participants truly desire to participate in the study and that they are not being coerced into doing so by family members.

\section{References}

1. Alzheimer's Disease International (ADI) (2015) World Alzheimer Report 2015, The Global Economic Impact of Dementia. Alzheimer's Disease International (ADI): $1-52$.

2. Dubroff JG, Nasrallah IM (2015) Will PET Amyloid Imaging Lead to Overdiagnosis of Alzheimer Dementia? Acad Radiol 22: 988-994.

3. Nobile H, Bergmann MM, Moldenhauer J, Borry P (2016) Participants accounts on their decision to join a cohort study with an attached biobank: a qualitative content analysis study within two german studies. J Empir Res Hum Res Ethics 11: 237-249.

4. Hallowell N, Cooke S, Crawford G, Lucassen A, Parker M, et al. (2010) An investigation of patients' motivations for their participation in genetics-related research. Journal of medical eth J Med Ethics 36: 37-45.

5. Lawrence V, Pickett J, Ballard C, Murray J (2014) Patient and carer views on participating in clinical trials for prodromal Alzheimer's disease and mild cognitive impairment. Int J Geriatr Psychiatry 29: 22-31.
6. Vanderschaeghe G, Schaeverbeke J, Vandenberghe R, Dierickx K (2017) Amnestic MCI patients' perspectives toward disclosure of amyloid PET results in a research context. Neuroethics 1-17.

7. Petersen RC, Doody R, Kurz A, Mohs RC, Morris JC, et al. (2001) Current concepts in mild cognitive impairment. Arch Neurol 58: 1985-1992.

8. ICH Expert Working Group (1996) Guideline for Good Clinical Practice E6 (ICH-GCP). ICH Harmonised Tripartite Guideline.

9. Hsieh HF, Shannon SE (2005) Three Approaches to Qualitative Content Analysis. Qual Health Res 15: 1277-1288.

10. Bazeley P (2007) Qualitative Data Analysis with NVivo. (2 ${ }^{\text {nd }}$ edn), SAGE Publications Ltd.

11. Steinsbekk KS, Solberg B (2012) Should genetic findings from genome research be reported back to the participants? Tidsskrift for den Norske lægeforening: tidsskrift for praktisk medicin, ny række 132: 2190-2193.

12. Ravitsky V, Wilfond BS (2016) Disclosing individual genetic results to research participants. The American journal of bioethics: AJOB 6: 8-17.

13. Wolf SM (2013) Return of individual research results and incidental findings: facing the challenges of translational science. Annual review of genomics and human genetics 14: 557-577.

14. Resnik DB (2011) Disclosure of individualized research results: a precautionary approach. Account Res 18: 382-397.

15. Burke W, Evans BJ, Jarvik GP (2014) Return of results: ethical and legal distinctions between research and clinical care. Am J Med Genet C Semin Med Genet 166C: 105-111.

16. Bredenoord AL, Kroes HY, Cuppen E, Parker M, Van Delden JJ (2011) Disclosure of individual genetic data to research participants: the debate reconsidered. Trends Genet 27: 41-47.

17. Shalowitz DI, Miller FG (2005) Disclosing individual results of clinical research: implications of respect for participants. JAMA 294: 737-740.

18. Viloria Jiménez MA, Chung Jaén M, Vigara García M, Barahonal Alvarez H (2013) Decision-making in older people with dementia. Reviews in Clinical Gerontology 23: 307-316.

19. Antoine P, Pasquier F (2013) Emotional and psychological implications of early AD diagnosis. Med Clin North Am 97: 459-475.

20. Defanti CA, Tiezzi A, Gasparini M, Gasperini M, Congedo M, et al. (2007) Ethical questions in the treatment of subjects with dementia Part I Respecting autonomy: awareness, competence and behavioural disorders. Neurol Sci 28: 216-231.

21. Prvulovic D, Hampel H (2011) Ethical considerations of biomarker use in neurodegenerative diseases-a case study of Alzheimer's disease. Progress in neurobiology 95: 517-519.

22. Christensen KD, Roberts JS, Uhlmann WR, Green RC (2011) Changes to perceptions of the pros and cons of genetic susceptibility testing after APOE genotyping for Alzheimer disease risk. Genet Med 13: 409-414. 